MATHEMATICS OF COMPUTATION

Volume 72, Number 243, Pages 1453-1471

S 0025-5718(03)01511-4

Article electronically published on March 26, 2003

\title{
SHORT UNIVERSAL GENERATORS VIA GENERALIZED RATIO-OF-UNIFORMS METHOD
}

\author{
JOSEF LEYDOLD
}

\begin{abstract}
We use inequalities to design short universal algorithms that can be used to generate random variates from large classes of univariate continuous or discrete distributions (including all log-concave distributions). The expected time is uniformly bounded over all these distributions for a particular generator. The algorithms can be implemented in a few lines of high level language code.
\end{abstract}

\section{INTRODUCTION}

In the last decade several approaches have been introduced for so-called universal (or black box) methods for generating nonuniform random variates. Recent papers propose methods where a hat function that approximates the respective probability density function or probability vector is constructed (see e.g., 1], 13], [2], [10, [17, 18]; [14, [15]). These methods have (extremely) fast marginal generation time, but require a setup step, which is expensive compared to the average cost of generating one random variate. Although this setup step can be made short at the price of a much higher marginal generation time (e.g., [11]), the resulting algorithms are rather complex.

Another approach by Devroye [6], on the other hand, uses universal inequalities that hold for every log-concave distribution. The algorithm for continuous random variates is based on the following proposition.

Theorem 1 (7], §VII.2.5, Theorem 2.4). If $f$ is a log-concave density with mode $\mu=0$ and $f(0)=1$, then writing $q$ for $F(0)$, where $F$ denotes the c.d.f. of the distribution, we have

$$
f(x) \leq \begin{cases}\min \left(1, e^{1-x /(1-q)}\right) & (x \geq 0) \\ \min \left(1, e^{1+x / q}\right) & (x<0) .\end{cases}
$$

The area under the bounding curve is 2 .

Received by the editor August 8, 2000.

2000 Mathematics Subject Classification. Primary 65C10; Secondary 65 U05.

Key words and phrases. Nonuniform random variates, universal method, ratio-of-uniforms method, transformed density rejection, discrete distributions, continuous distributions, logconcave distributions, $T$-concave distributions.

This work was supported by the Austrian Science Foundation (FWF), project no. P12805MAT.

(C)2003 American Mathematical Society 
If $F(\mu)$ is not known, a modified universal hat exists with area 4 (see [7], $\S$ VII.2.3). In both cases these universal hats are not optimal. [6] derives the properties of the optimal hat and provides a (rather expensive) generator for the corresponding distribution. The areas below the optimal bounding curves are $\pi^{2} / 6$ and $\pi^{2} / 3$, respectively, i.e., about $18 \%$ better. Algorithms that utilize this theorem can be found in 7]. [8] gives an analogous result for discrete log-concave distributions.

In 19 the ratio-of-uniforms method is used to derive more general inequalities that were used to compile faster and simpler algorithms that work for a larger class of distributions, including all log-concave distributions. As in Devroye's algorithm and in opposition to other black-box algorithms, hardly any setup step is required. Thus it is superior in the changing parameter case.

In this paper this approach is extended. We introduce universal bounding curves based on the generalized ratio-of-uniforms method by [21]. The new algorithms are still universal, and the expected numbers of uniform random numbers are uniformly bounded for each of these algorithms. They are applicable to a large class of socalled $T$-concave distributions [13] at the expense of higher marginal generation times. For the subclass of heavy-tailed $T$-concave distributions these inequalities can be used to compile even faster algorithms. Therefore they complement the universal algorithm introduced by [5] (see also [7, §VII.3.2]) that uses moments of monotone distributions.

The new bounding curves can also be used to derive general upper bounds for probability density functions of $T$-concave distributions. These are optimal in the sense that constructing any better majorizing function requires more information about such a density function.

\section{RATIO-OF-UNIFORMS AND T-CONCAVE DISTRIBUTIONS}

2.1. Ratio-of-uniforms. The ratio-of-uniforms method introduced by [16 is a flexible method that can be adjusted to a large variety of distributions. It has become a popular transformation method to generate nonuniform random variates, since it results in exact, efficient, fast and easy to implement algorithms. It is based on the following (slightly modified) theorem.

Theorem 2 (16]). Let $f(x)$ be a positive integrable function with support $\left(x_{0}, x_{1}\right)$ not necessarily finite. If $(V, U)$ is uniformly distributed in

$$
\mathcal{A}=\mathcal{A}(f)=\left\{(v, u): 0<u \leq \sqrt{f(v / u+\mu)}, x_{0}<v / u+\mu<x_{1}\right\},
$$

then $X=V / U+\mu$ has probability density function $f(x) / \int f$.

For sampling random points uniformly distributed in $\mathcal{A}$, rejection from a convenient enveloping region is used, usually from the minimal bounding rectangle. Its boundaries have to be calculated analytically for each distribution. If $\mathcal{A}$ is convex, however, it is easy to construct a universal bounding rectangle without computing these boundaries (see below).

2.2. $\boldsymbol{T}$-concave distributions. A probability density function $f(x)$ is called $T$ concave if there exists a monotonically increasing, differentiable transformation $T(x)$ such that $T(f(x))$ is concave. A distribution is called $T$-concave if its probability density function is $T$-concave. The transformed density rejection method is an acceptance/rejection technique that uses $T$-concavity of a large class of distributions to construct hat function and squeeze for the density automatically (see [13] or 
[10] for details). [13] suggests the family $T_{c}$ of transformations with $T_{0}(x)=\log (x)$ and $T_{c}(x)=-x^{c}$ for $-1<c<0$. $(c \leq-1$ is possible for densities with compact domain.) If a function $f$ is $T_{c_{1}}$-concave, then it is also $T_{c}$-concave for every $c<c_{1}$ [13]. An equivalent transformation is $\tilde{T}_{c}(x)=\left(x^{c}-1\right) / c$, for $c \neq 0$, and $\tilde{T}_{0}(x)=\log (x)$, also known as a Box-Cox transformation. It is continuous in $c$ for $c=0$.

[20] and [9] have clarified the relationship of the ratio-of-uniforms method to the ordinary acceptance/rejection method. It can be viewed as rejection from a tablemountain shaped density (see Figure 21). [17] has shown a deeper connection to transformed density rejection. Moreover, a full characterization of all distributions with convex region $\mathcal{A}$ is derived.

Theorem $3([17]) . \mathcal{A}(f)$ is convex if and only if $f(x)$ is T-concave with transformation $T(x)=-1 / \sqrt{x}$, i.e., if and only if $-1 / \sqrt{f(x)}$ is a concave function.

Notice that this class of $T$-concave distributions includes all log-concave distributions.

2.3. Universal inequalities. Using the convexity of $\mathcal{A}(f)$ for this class of distributions, we arrive at the following proposition (see Figure 1).

Theorem 4 ([19, Theorems 4 and 5). For a distribution with density $f$, c.d.f. $F$ and mode $\mu$ let

$$
\begin{aligned}
& \mathcal{R}=\left\{(v, u): v_{l} \leq v \leq v_{r}, 0 \leq u \leq u_{m}\right\} \\
& \mathcal{Q}=\left\{(v, u):-v_{m} \leq v \leq v_{m}, 0 \leq u \leq u_{m}\right\}
\end{aligned}
$$

and

$$
\mathcal{S}=\left\{(v, u): v_{l} u \leq u_{m} v \leq v_{r} u, v_{l}\left(u_{m}-u\right) \leq u_{m} v \leq v_{r}\left(u_{m}-u\right)\right\}
$$

where

$$
\begin{array}{ll}
u_{m}=\sqrt{f(\mu)}, & v_{l}=-F(\mu) v_{m}, \\
v_{m}=\left(\int f\right) / \sqrt{f(\mu)}, & v_{r}=(1-F(\mu)) v_{m} .
\end{array}
$$

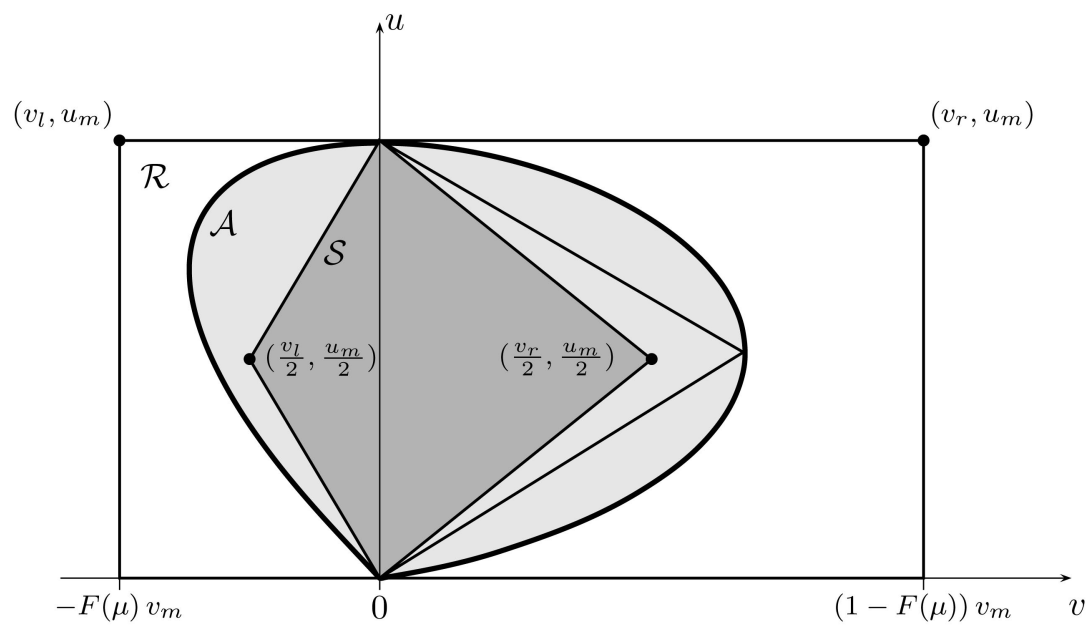

Figure 1. $\mathcal{A}$ and universal bounding rectangle $\mathcal{R}$ and squeeze $\mathcal{S}$ for gamma(3) distribution. 

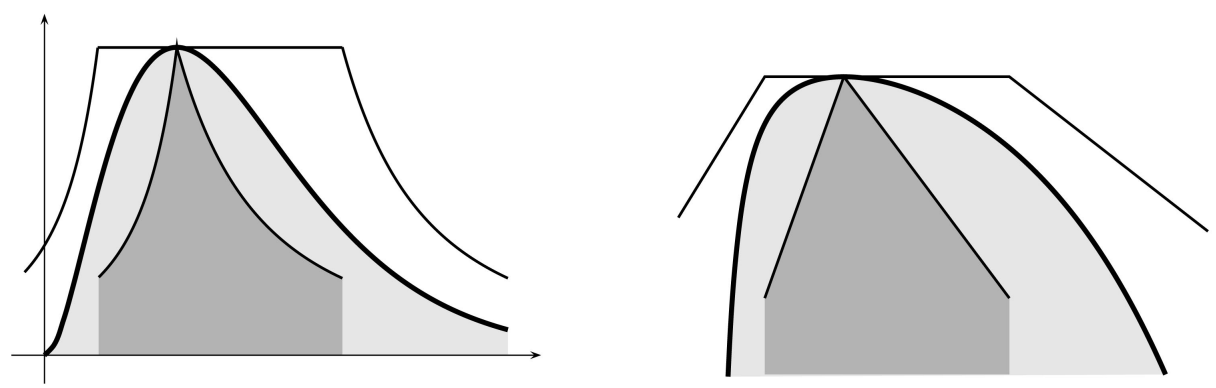

Figure 2. Universal hat and squeeze for gamma(3) distribution. Original scale (l.h.s.) and transformed scale (r.h.s.)

Then for any $T$-concave distribution with $T(x)=-1 / \sqrt{x}$ we find that

$$
\mathcal{S} \subset \mathcal{A} \subset \mathcal{R} \subset \mathcal{Q} .
$$

Moreover,

$$
2|\mathcal{S}|=|\mathcal{A}|=\frac{1}{2}|\mathcal{R}|=\frac{1}{4}|\mathcal{Q}|,
$$

where $|\mathcal{A}|$ denotes the area of $\mathcal{A}$.

Notice that only the knowledge of $F(\mu)$ is required, e.g., $F(\mu)=\frac{1}{2}$ for symmetric distributions, or $F(\mu)=0$ for monotonically decreasing densities. Moreover, $\mathcal{Q}$ does not depend on $F(\mu)$ at all. By equation (7) the rejection constant of an algorithm that uses Theorem 4 is 2 when $F(\mu)$ is known, and 4 otherwise.

Theorem 3 is proved by means of an appropriate transformation (see Theorem 6 below). This transformation can be used to derive universal upper and lower bounds for $T$-concave distributions [19, Theorem 7], see Figure 2]

\section{A generalization}

Theorem 5 (21]). Let $f(x)$ be a positive integrable function with support $\left(x_{0}, x_{1}\right)$ not necessarily finite. Let $g$ be a strictly increasing differentiable function on $[0, \infty)$ such that $g(0)=0$, and let $k$ and $\mu$ be constants. Suppose the pair of variables $(V, U)$ is uniformly distributed over the region

(8) $\mathcal{A}_{g}=\mathcal{A}_{g}(f)=\left\{(v, u): 0<u \leq g^{-1}\left[k f\left(v / g^{\prime}(u)+\mu\right)\right], x_{0}<v / g^{\prime}(u)+\mu<x_{1}\right\}$.

Then $X=V / g^{\prime}(U)+\mu$ has probability density function $f(x) / \int f$.

[21] suggested power functions $g_{r}(u)=u^{r+1} /(r+1), r>0$ and $k=1 /(r+1)$. Then equation (8) becomes

$$
\mathcal{A}_{r}=\mathcal{A}_{r}(f)=\left\{(v, u): 0<u \leq \sqrt[r+1]{f\left(v / u^{r}+\mu\right)}, x_{0}<v / u^{r}+\mu<x_{1}\right\} .
$$

The minimal bounding rectangle for $\mathcal{A}_{r}$ is given by (see [21])

$$
\mathcal{R}_{\mathrm{mbr}}=\left\{(v, u): v^{-} \leq v \leq v^{+}, 0 \leq u \leq u_{m}\right\}
$$


where

$$
\begin{aligned}
& u_{m}=\sup _{x_{0}<x<x_{1}} \sqrt[r+1]{f(x)}, \\
& v^{-}=\inf _{x_{0}<x<x_{1}}(x-\mu)[f(x)]^{r /(r+1)}, \\
& v^{+}=\sup _{x_{0}<x<x_{1}}(x-\mu)[f(x)]^{r /(r+1)} .
\end{aligned}
$$

There also exists a generalization of Theorem 3 . Consider the following regions:

$$
\begin{aligned}
& \mathcal{B}_{r}=\mathcal{B}_{r}(f)=\left\{(v, w): 0<w \leq[f(v / w+\mu)]^{r /(r+1)}, x_{0}<v / w+\mu<x_{1}\right\} \\
& \mathcal{G}=\mathcal{G}(f)=\left\{(x, y): 0<y \leq f(x), x_{0}<x<x_{1}\right\} \\
& \mathcal{T}_{c}=\mathcal{T}_{c}(f)=\left\{(x, y): 0<y \leq-(f(x))^{c}, x_{0}<x<x_{1}\right\}
\end{aligned}
$$

$\mathcal{G}(f)$ is the region between the graph of $f$ and the $x$-axis. $\mathcal{T}_{c}(f)$ is the region below the graph of the transformed density $T_{c}(f(x))$, see Figure 3 .

Theorem 6. $\mathcal{B}_{r}(f)$ is convex if and only if $f(x)$ is $T_{c}$-concave with $c=-\frac{r}{r+1}$.

Transformations between the above regions play a crucial role for the proof of this theorem as well as for the further developement of the theory of this type of generation methods. Consider the transformations

$$
\begin{array}{lll}
\Phi_{\mathcal{A B}}: & \mathbb{R} \times \mathbb{R}^{+} \rightarrow \mathbb{R} \times \mathbb{R}^{+}, & (V, U) \mapsto(V, W)=\left(V, U^{r}\right), \\
\Phi_{\mathcal{B G}}: & \mathbb{R} \times \mathbb{R}^{+} \rightarrow \mathbb{R} \times \mathbb{R}^{+}, & (V, W) \mapsto(X, Y)=\left(V / W+\mu, W^{(r+1) / r}\right), \\
\Phi_{\mathcal{G T}}: & \mathbb{R} \times \mathbb{R}^{+} \rightarrow \mathbb{R} \times \mathbb{R}^{-}, & (X, Y) \mapsto(X, Z)=\left(X,-Y^{c}\right) .
\end{array}
$$

Notice that $\Phi_{\mathcal{A B}}$ maps $\mathcal{A}_{r}$ one-to-one onto $\mathcal{B}_{r}$. Analogously for $\Phi_{\mathcal{B G}}$ and $\Phi_{\mathcal{G} \mathcal{T}}$.
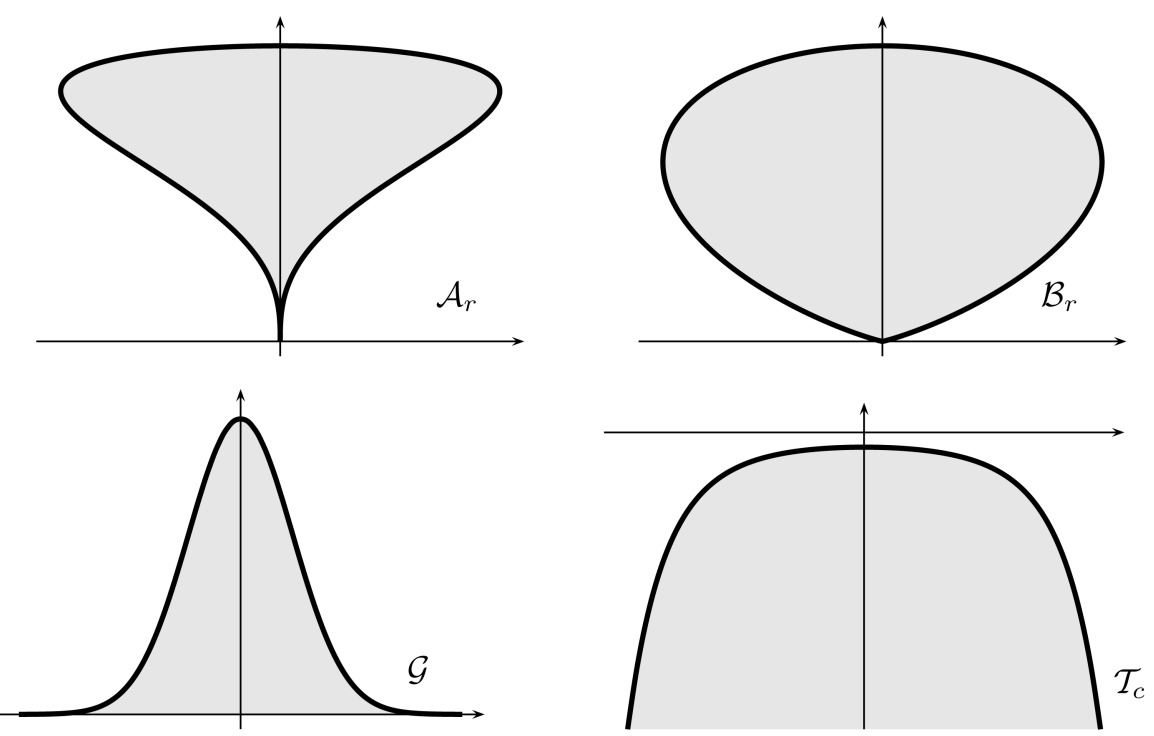

FiguRE 3. $\mathcal{A}_{r}, \mathcal{B}_{r}, \mathcal{G}$, and $\mathcal{T}_{c}$ for the normal distribution with $r=3$ and $c=-3 / 4$. Notice that $\mathcal{B}_{r}$ is convex if and only if $\mathcal{T}_{c}$ is convex (Theorem 6). 
Proof of the Theorem. Let $c=-\frac{r}{r+1}$. The transformation

$$
\begin{aligned}
\Phi_{\mathcal{B} \mathcal{T}}=\Phi_{\mathcal{G} \mathcal{T}} \circ \Phi_{\mathcal{B G}}: & \mathbb{R} \times \mathbb{R}^{+} \rightarrow \mathbb{R} \times \mathbb{R}^{-}, \\
& (V, W) \mapsto\left(\frac{V}{W}+\mu,-W^{c \frac{r+1}{r}}\right)=\left(\frac{V}{W}+\mu,-\frac{1}{W}\right)
\end{aligned}
$$

maps $\mathcal{B}_{r}$ one-to-one onto $\mathcal{T}_{c}$. Notice that $f$ is $T_{c}$-concave if and only if $\mathcal{T}_{c}$ is convex. Thus it remains to show that $\mathcal{B}_{r}$ is convex if and only if $\mathcal{T}_{c}$ is convex, and consequently that straight lines remain straight lines under the transformation $\Phi_{\mathcal{B T}}$.

Let $a x+b y=d$ be a straight line in $\mathcal{T}_{c}$. Then $a(v / w+\mu)-b / w=d$ and $a v+(1-d) w=b$, i.e., a straight line in $\mathcal{B}_{r}$. Analogously a straight line $a v+b w=d$ in $\mathcal{B}_{r}$ is mapped onto the line $a x+d y=a \mu-b$ in $\mathcal{T}_{c}$.

The transformation $\Phi_{\mathcal{A G}}=\Phi_{\mathcal{B G}} \circ \Phi_{\mathcal{A B}}:(V, U) \mapsto(X, Y)=\left(V / U^{r}+\mu, U^{r+1}\right)$ has Jacobian $r+1$, and thus Theorem $[5$ follows for the power function $g(u)=$ $u^{r+1} /(r+1)$. (For an arbitrary function $g(u)$ the proof is completely analogous.) Moreover,

$$
\left|\mathcal{A}_{r}(f)\right|=\int f /(r+1) .
$$

Remark. For $r=1$ we have $\mathcal{B}_{1}=\mathcal{A}_{1}=\mathcal{A}$ (equations (12), (9) and (2)). Thus Theorems 2 and 3 are corollaries of the respective Theorems [5] and 6 .

3.1. A universal envelope. Now let $f(x)$ be a $T_{c}$-concave density function with $c=-\frac{r}{r+1}$ and mode $\mu$. Define $\mathcal{A}_{r}^{+}=\left\{(v, u) \in \mathcal{A}_{r}: v \geq 0\right\}, \mathcal{A}_{r}^{-}=\left\{(v, u) \in \mathcal{A}_{r}: v \leq\right.$ $0\}$, and analogously $\mathcal{B}_{r}^{+}$and $\mathcal{B}_{r}^{-}$. By Theorem $6, \mathcal{B}_{r}^{+}$is convex and thus contains the triangle with vertices at $(0,0),\left(0, u_{m}^{r}\right)$ and the extremal point with coordinates $\left(v^{+}, u_{e}^{r}\right)$, where $u_{e} \geq 0$ (see Figure 4 ). Then transformation $\Phi_{\mathcal{A B}}^{-1}$ maps this triangle into a three-side region in $\mathcal{A}_{r}$ of area

$$
\int_{0}^{v^{+}}\left(\left[u_{m}^{r}+v\left(u_{e}^{r}-u_{m}^{r}\right) / v^{+}\right]^{1 / r}-\left[v u_{e}^{r} / v^{+}\right]^{1 / r}\right) d v=v^{+} u_{m}^{r} \frac{r}{r+1} \frac{u_{e}-u_{m}}{u_{e}^{r}-u_{m}^{r}} .
$$

By the convexity of $\mathcal{B}_{r}^{+}$this cannot exceed $\left|\mathcal{A}^{+}\right|$, and consequently

$$
v^{+} \leq\left|\mathcal{A}^{+}\right| \frac{1}{u_{m}^{r}} \frac{r+1}{r} \frac{u_{e}^{r}-u_{m}^{r}}{u_{e}-u_{m}}=\left|\mathcal{A}^{+}\right| \frac{1}{u_{m}} \frac{r+1}{r} \frac{\left(u_{e} / u_{m}\right)^{r}-1}{\left(u_{e} / u_{m}\right)-1} .
$$
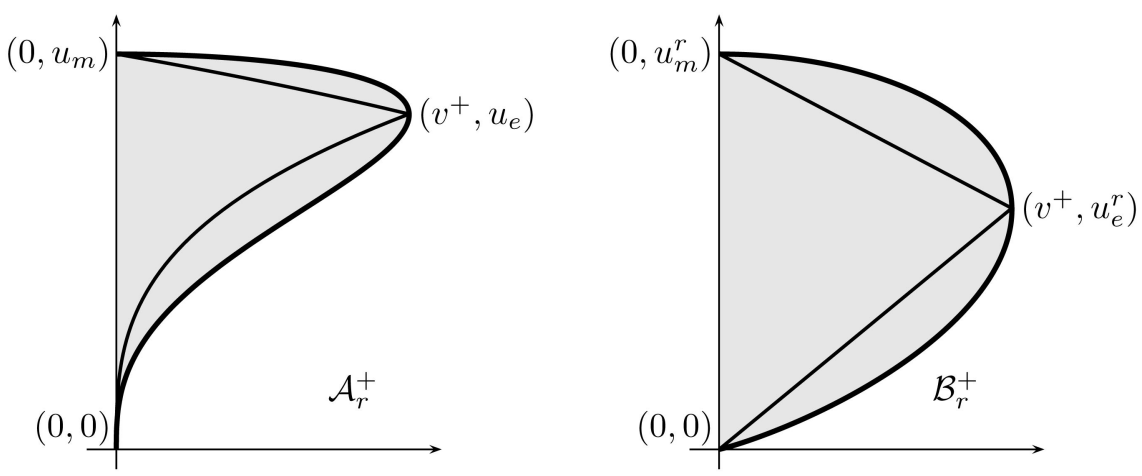

Figure 4 . The region $\mathcal{B}_{r}^{+}$with enclosed triangle with vertices at $(0,0),\left(0, u_{m}^{r}\right)$ and extremal point $\left(v^{+}, u_{e}^{r}\right)$ (r.h.s.). $\mathcal{A}_{r}^{+}$and the corresponding enclosed region are on the l.h.s. 
Using (14) and the fact that $\left|\mathcal{A}^{+}\right|=(1-F(\mu))|\mathcal{A}|$, we arrive at

$$
v^{+} \leq(1-F(\mu))\left(\int f\right) \frac{1}{r u_{m}} \frac{\left(u_{e} / u_{m}\right)^{r}-1}{\left(u_{e} / u_{m}\right)-1} .
$$

An analogous (lower) bound can be derived for $v^{-}$. Thus we have the following universal envelopes.

Theorem 7. Let $f(x)$ be a $T_{c}$-concave density function with $c=-\frac{r}{r+1}, r>0$, and mode $\mu$. Let $F$ denote the c.d.f. of the distribution and let

$$
\begin{aligned}
& \mathcal{R}_{r}=\left\{(v, u): v_{l}(u) \leq v \leq v_{r}(u), 0 \leq u \leq u_{m}\right\}, \\
& \mathcal{Q}_{r}=\left\{(v, u):-v_{m}(u) \leq v \leq v_{m}(u), 0 \leq u \leq u_{m}\right\},
\end{aligned}
$$

where

$$
\begin{array}{ll}
u_{m}=\sqrt[r+1]{f(\mu)}, & v_{m}(u)=\left(\int f\right) /\left(r u_{m}\right) \frac{\left(u / u_{m}\right)^{r}-1}{\left(u / u_{m}\right)-1}, \\
v_{l}(u)=-F(\mu) v_{m}(u), & v_{r}(u)=(1-F(\mu)) v_{m}(u) .
\end{array}
$$

Then $\mathcal{A}_{r} \subset \mathcal{R}_{r} \subset \mathcal{Q}_{r}$ and

$$
\left|\mathcal{R}_{r}\right|=\frac{r+1}{r}(\gamma+\psi(r+1))\left|\mathcal{A}_{r}\right| \quad \text { and } \quad\left|\mathcal{Q}_{r}\right|=2\left|\mathcal{R}_{r}\right|,
$$

where $\gamma=0.577216 \ldots$ denotes Euler's (gamma) constant and $\psi(z)=\Gamma^{\prime}(z) / \Gamma(z)$ is the digamma function (also known as Euler's psi function). The envelopes $\mathcal{R}_{r}$ and $\mathcal{Q}_{r}$ are optimal.

Proof. By equation (17) every point $(v, u) \in \mathcal{A}_{r}$ satisfies the respective conditions for $\mathcal{R}_{r}$ and $\mathcal{Q}_{r}$ in equation (18). Consequently $\mathcal{A}_{r} \subset \mathcal{R}_{r} \subset \mathcal{Q}_{r}$. It remains to verify (20). From (19) and (14) we get

$$
\begin{aligned}
\left|\mathcal{R}_{r}\right| & =\left|\mathcal{R}_{r}^{+}\right|+\left|\mathcal{R}_{r}^{-}\right|=\int_{0}^{u_{m}}\left(\int f\right) /\left(r u_{m}\right) \frac{\left(u / u_{m}\right)^{r}-1}{\left(u / u_{m}\right)-1} d u \\
& =\int_{0}^{u_{m}}\left|\mathcal{A}_{r}\right| \frac{r+1}{r} \frac{\left(u / u_{m}\right)^{r}-1}{\left(u / u_{m}\right)-1} \frac{1}{u_{m}} d u \\
& =\left|\mathcal{A}_{r}\right| \frac{r+1}{r} \int_{0}^{1} \frac{z^{r}-1}{z-1} d z=\left|\mathcal{A}_{r}\right| \frac{r+1}{r}(\gamma+\psi(r+1)),
\end{aligned}
$$

where the last equality follows from formula 8.361(7) in [12, p.952].

Figure 5 illustrates the situation for the Cauchy distribution and $r=3$. Figure 6 plots the ratio of $\left|\mathcal{R}_{r}\right| /\left|\mathcal{A}_{r}\right|$ against $r$. Generating points uniformly distributed over $\mathcal{R}_{r}$ requires an appropriate enveloping region. Transformed density rejection can be used to construct such a region. We need the following two lemmata.

Lemma 8 ([3]). $\frac{x-1}{x^{r}-1}$ is convex for $r \geq 1$ and $x \in[0,1)$.

Proof. The proposition is trivial for $r=1$. Thus assume $r>1$. By a straightforward computation we obtain

$$
\left((x-1) /\left(x^{r}-1\right)\right)^{\prime \prime}=\frac{r x^{r-2}}{\left(x^{r}-1\right)^{3}} \cdot\left[(r-1)\left(x^{r+1}-1\right)-(r+1)\left(x^{r}-x\right)\right],
$$

which is greater than or equal to 0 if and only if the second factor is not greater than 0 , i.e., if and only if

$$
A(x)=\frac{x^{r+1}-1}{r+1}-\frac{x^{r}-x}{r-1} \leq 0 .
$$


Notice that $A^{\prime}(x)=x^{r}-\frac{r x^{r-1}-1}{r-1}$ and $A^{\prime \prime}(x)=r(x-1) x^{r-2} \leq 0$. Consequently $A^{\prime}(x)$ is monotonically decreasing and, since $A^{\prime}(1)=0, A^{\prime}(x) \geq 0$. Hence $A(x)$ is monotonically increasing and, since $A(1)=0, A(x) \leq 0$, and the proposition follows.

Lemma 9. Let $\tau(x)=-\frac{x-1}{x^{r}-1}$, and let $a=\tau\left(x_{0}\right)-x_{0} \tau^{\prime}\left(x_{0}\right)$ and $b=\tau^{\prime}\left(x_{0}\right)$ for an $x_{0} \in(0,1)$. Assume $r \geq 1$. Then $-\frac{1}{a+b x} \geq \frac{x^{r}-1}{x-1}$ for all $x \in[0,1]$.

Proof. By Lemma $8 \tau(x)$ is concave. Thus for the tangent in $x_{0}$ we find that $a+b x \geq \tau(x)$, for all $x \in[0,1)$. Now notice that

$$
\tau^{\prime}(x)=\left(x^{r}-1\right)^{-2} \cdot\left(1+r(x-1) x^{r-1}-x^{r}\right),
$$

and

$$
\lim _{x \rightarrow 1} \tau^{\prime}(x)=(r-1) /(2 r) \geq 0,
$$

by l'Hospital's rule. Thus, by the concavity of $\tau(x), \tau^{\prime}(x)>0$ for all $x \in(0,1)$, i.e., $b>0$. Hence $a+b x \leq a+b$. Notice that $a+b$ is the tangent of $\tau$ with construction point $x_{0}$ evaluated at $x=1$. By the concavity of $\tau, a+b$ is maximized in $x_{0}=0$. Hence $\tau(x) \leq a+b x<a+b \leq \tau(0)+\tau^{\prime}(0)=0$. Consequently $-1 /(a+b x) \geq-1 / \tau(x)$, and the proposition follows.

The following enveloping region for $\mathcal{R}_{r}$ has been suggested by 3 .

Theorem 10. Let $f(x)$ be a $T_{c}$-concave probability density function with $c=-\frac{r}{r+1}$, $r \geq 1$, and mode $\mu$. Let $F$ denote the c.d.f. of the distribution. Let

$$
x_{r}=1-2.187 /(r+5-1.28 / r)^{0.9460}
$$

and denote $a_{r}=\tau\left(x_{r}\right)-x_{r} \tau^{\prime}\left(x_{r}\right)$ and $b_{r}=\tau^{\prime}\left(x_{r}\right)$, where $\tau(x)=-(x-1) /\left(x^{r}-1\right)$. Let

$$
\begin{aligned}
& \tilde{\mathcal{R}}_{r}=\left\{(v, u): \tilde{v}_{l}(u) \leq v \leq \tilde{v}_{r}(u), 0 \leq u \leq u_{m}\right\}, \\
& \tilde{\mathcal{Q}}_{r}=\left\{(v, u):-\tilde{v}_{m}(u) \leq v \leq \tilde{v}_{m}(u), 0 \leq u \leq u_{m}\right\},
\end{aligned}
$$

where

$$
\begin{array}{ll}
u_{m}=\sqrt[r+1]{f(\mu)}, & \tilde{v}_{m}(u)=\left(\int f\right) /\left(r u_{m}\right) \frac{-1}{a_{r}+b_{r}\left(u / u_{m}\right)}, \\
\tilde{v}_{l}(u)=-F(\mu) \tilde{v}_{m}(u), & \tilde{v}_{r}(u)=(1-F(\mu)) \tilde{v}_{m}(u) .
\end{array}
$$

Then $\mathcal{A}_{r} \subset \tilde{\mathcal{R}}_{r} \subset \tilde{\mathcal{Q}}_{r}$ and

$$
\left|\tilde{\mathcal{R}}_{r}\right|=\frac{r+1}{r} \frac{1}{b_{r}} \log \left(\frac{a_{r}}{a_{r}+b_{r}}\right)\left|\mathcal{A}_{r}\right| \quad \text { and } \quad\left|\tilde{\mathcal{Q}}_{r}\right|=2\left|\tilde{\mathcal{R}}_{r}\right| .
$$

Proof. $\mathcal{A}_{r}(f) \subset \mathcal{R}_{r} \subseteq \tilde{\mathcal{R}}_{r}$ follows immediately from Theorem[7] and Lemma 9, To verify (24) we compute

$$
\begin{aligned}
\left|\tilde{\mathcal{R}}_{r}\right| & =\left|\tilde{\mathcal{R}}_{r}^{+}\right|+\left|\tilde{\mathcal{R}}_{r}^{-}\right|=\int_{0}^{u_{m}}\left|\mathcal{A}_{r}\right| \frac{r+1}{r} \frac{1}{u_{m}} \frac{-1}{a_{r}+b_{r}\left(u / u_{m}\right)} d u \\
& =\left|\mathcal{A}_{r}\right| \frac{r+1}{r} \int_{0}^{1} \frac{1}{a_{r}+b_{r} z} d z=\left|\mathcal{A}_{r}\right| \frac{r+1}{r} \frac{1}{b_{r}} \log \left(a_{r} /\left(a_{r}+b_{r}\right)\right) .
\end{aligned}
$$

Remark. The parameters for $x_{r}$ in equation (21) have been found by minimizing the area of $\tilde{\mathcal{R}}_{r}$. Numerical computations show that $\left|\tilde{\mathcal{R}}_{r}\right|<1.13\left|\mathcal{R}_{r}\right|$. 
To generate points uniformly in $\tilde{\mathcal{R}}_{r}$ we have to generate random variates with density proportional to $h(x)=-1 /(a+b x)$ on $[0,1]$. This can be done by inversion. Notice that $a+b x<0$ for all $x \in[0,1]$. The c.d.f. is given by

$$
H(x)=\frac{\log (a /(a+b x))}{\log (a /(a+b))}
$$

and thus we find for the inverse of the c.d.f.

$$
H^{-1}(u)=\frac{a}{b}(\exp (-u \log (a /(a+b)))-1)
$$

Using the above results, we can compile algorithm GSROUC.

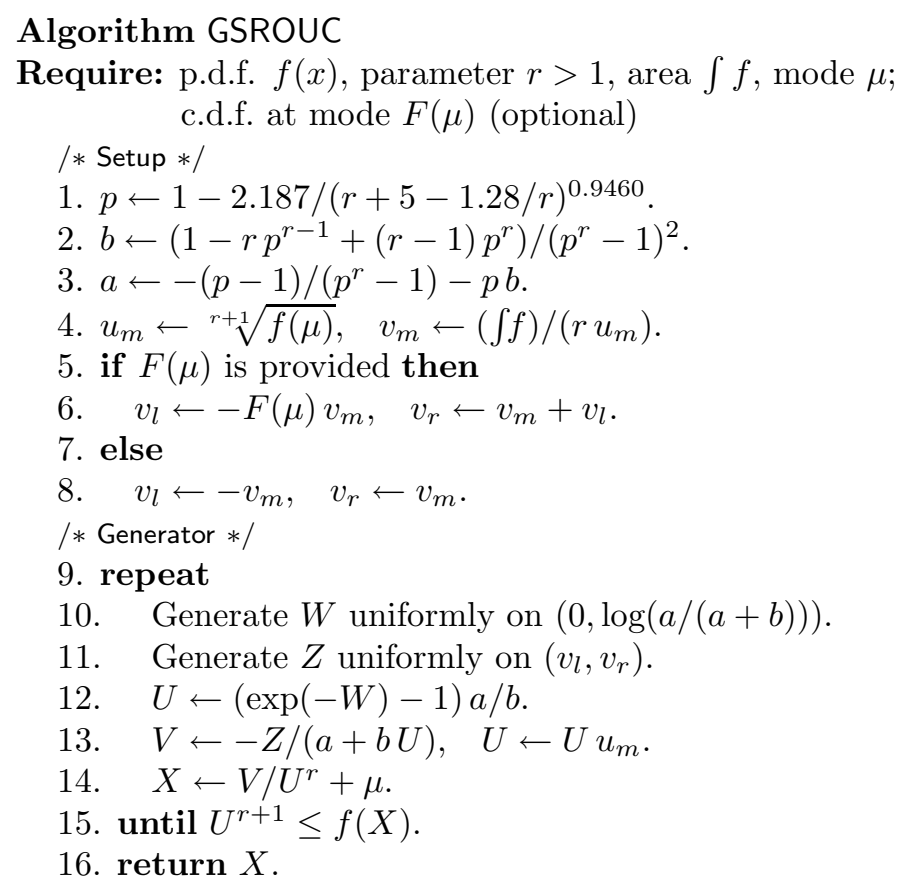

Remark. There is no need for an algorithm for $r<1$, since then the much simpler and faster algorithm that applies Theorem 4 can be used (see algorithm SROU in [19]).

Remark. It should be noted that for $r \rightarrow 0$ we have $c \rightarrow 0$ and $T_{c} \rightarrow \log$. Moreover,

$$
\lim _{r \rightarrow 0} \frac{r+1}{r}(\gamma+\psi(r+1))=\pi^{2} / 6
$$

The optimal algorithm of [6] (see remark after Theorem 11) is thus a limit case of the new method. However, this is of purely theoretical interest, since the regions $\mathcal{A}_{r}$ become long and skinny when $r$ tends to 0 .

3.2. A universal squeeze. Analogously to Theorem 4 there exists an (optimal) squeeze for $\mathcal{A}_{r}$. Figure 5 illustrates the situation for the Cauchy distribution and $r=3$.

Theorem 11. Let $f(x)$ be a $T_{c}$-concave probability density function with $c=-\frac{r}{r+1}$, $r>0$, and mode $\mu$. If $F(\mu)$ is given, where $F$ denotes the c.d.f. of the distribution, 
then there exists a set $\mathcal{S}_{r}=\mathcal{S}_{r}(f)$ such that $\mathcal{S}_{r} \subset \mathcal{A}_{r}(f)$. We have $(V, U) \in \mathcal{S}_{r}$ if and only if either

$$
0 \leq \frac{V}{U^{r}} \leq \frac{\bar{v}_{r}}{u_{m}^{r}} \quad \text { and } \quad U^{r} \bar{v}_{r}+V r u_{m}^{r} \leq \bar{v}_{r} u_{m}^{r}
$$

or

$$
0 \geq \frac{V}{U^{r}} \geq \frac{\bar{v}_{l}}{u_{m}^{r}} \quad \text { and } \quad U^{r} \bar{v}_{l}+V r u_{m}^{r} \geq \bar{v}_{l} u_{m}^{r} .
$$

where $u_{m}$ is as defined in Theorem 7 and $\bar{v}_{r}=(1-F(\mu))\left(\int f\right) / u_{m}$ and $\bar{v}_{l}=$ $-F(\mu)\left(\int f\right) / u_{m}$. Moreover,

$$
\left|\mathcal{S}_{r}\right|=\left|\mathcal{A}_{r}\right|\left(1-1 /(r+1)^{1 / r}\right) .
$$

Proof. By Theorem $6, \mathcal{B}_{r}(f)$ is convex. Let $\mathcal{S}_{r}$ denote the universal squeeze region (which might be empty) and let $\mathcal{C}=\Phi_{\mathcal{A B}}\left(\mathcal{S}_{r}\right)$ denote the corresponding region in $\mathcal{B}_{r}$. Assume that $\mathcal{A}_{r}^{+} \neq \emptyset$. Let $\Delta$ be the triangle defined by the set of all points $\left(v, u^{r}\right)$ that satisfy the inequalities $v \geq 0, v / u^{r} \leq \bar{v}_{r} / u_{m}^{r}$ and $u^{r} \bar{v}_{r}+v r u_{m}^{r} \leq \bar{v}_{r} u_{m}^{r}$. Its vertices are $(0,0),\left(0, u_{m}^{r}\right)$ and $\left(\bar{v}_{r} /(r+1), u_{m}^{r} /(r+1)\right)$. Define $\mathcal{C}^{+}=\{(v, u) \in$ $\mathcal{C}: v \geq 0\}$ and $\mathcal{R}_{r}^{+}=\left\{(v, u) \in \mathcal{R}_{r}: v \geq 0\right\}$. Every straight line through a point $\left(V, U^{r}\right)$ with $(V, U) \in \mathcal{R}_{r}^{+} \backslash \mathcal{A}_{r}^{+}$that does not intersect $\mathcal{B}_{r}^{+}$is transformed by $\Phi_{\mathcal{A B}}^{-1}$ into a curve that splits $\mathcal{R}_{r}^{+}$into two parts such that (i) $\mathcal{A}_{r}^{+}$and the edge $(0,0)\left(0, u_{m}\right)$ are completely contained in the left-hand part, and (ii) the area of the left-hand part must be at least $\left|\mathcal{A}_{r}^{+}\right| . \mathcal{S}_{r}^{+}$is then the intersection of the left-hand parts of all such curves. Consequently $\mathcal{C}^{+}$must be contained in the triangles with respective vertices at $(0,0),\left(0, u_{m}^{r}\right)$ and $\left(v_{r}(0), 0\right)$, and $(0,0),\left(0, u_{m}^{r}\right)$ and $\left(v_{r}\left(u_{m}\right), u_{m}^{r}\right)$, where $v_{r}(u)$ is the bounding curve for $\mathcal{R}$ given in equation (19). Notice that $v_{r}\left(u_{m}\right)=\bar{v}_{r}$ and $v_{r}(0)=\bar{v}_{r} / r$, and thus the respective right-hand vertices are $\left(\bar{v}_{r} / r, 0\right)$ and $\left(\bar{v}_{r}, u_{m}^{r}\right)$. Since the intersection of these triangles is given by $\Delta$, we have $\Delta \supseteq \mathcal{C}^{+}$. Now notice that $\mathcal{C}^{+}$is convex, because it is the intersection of triangles. It remains to show that the vertex $\left(\bar{v}_{r} /(r+1), u_{m}^{r} /(r+1)\right) \in \mathcal{C}^{+}$, since then $\Delta \subseteq \mathcal{C}^{+}$. A straight line through $(a, 0)$ and $\left(b, u_{m}^{r}\right)$ is given by

$$
v=w / u_{m}^{r}(b-a)+a,
$$

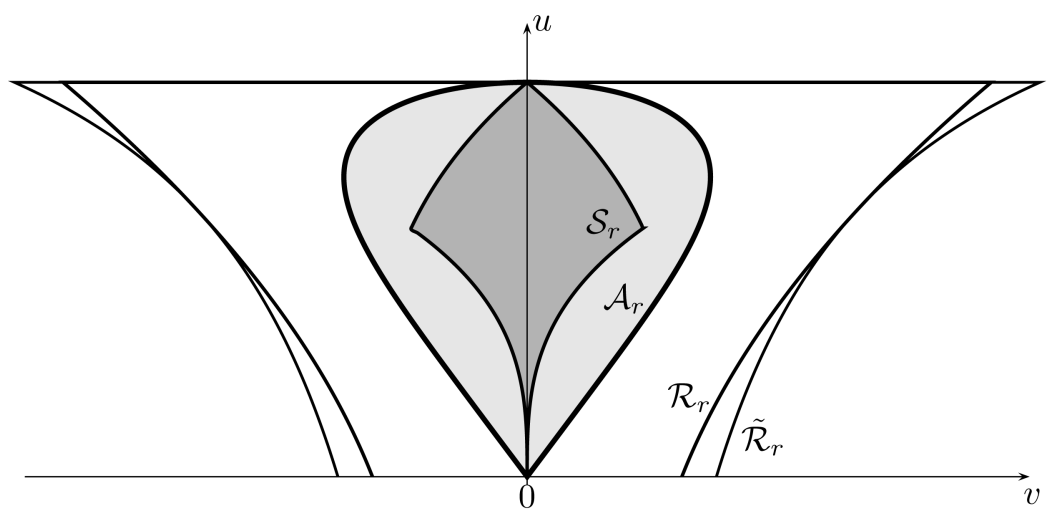

FiguRE 5. $\mathcal{A}_{r}$, universal bounding envelopes $\mathcal{R}_{r}$ and $\tilde{\mathcal{R}}_{r}$, and universal squeeze $\mathcal{S}_{r}$ for the Cauchy distribution $(r=3)$. 


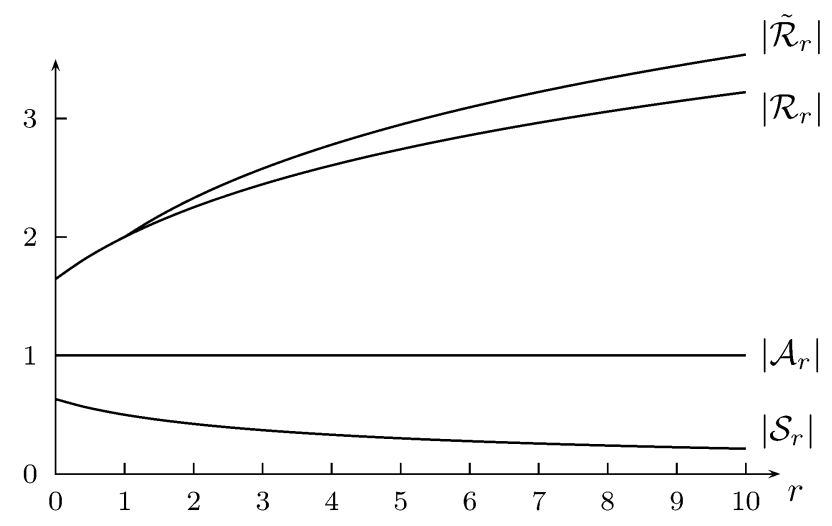

FiguRe 6. Respective ratios of $\left|\tilde{\mathcal{R}}_{r}\right|,\left|\mathcal{R}_{r}\right|$ and $\left|\mathcal{S}_{r}\right|$ to $\left|\mathcal{A}_{r}\right|$ as functions of $r$. (Notice that $\tilde{\mathcal{R}}_{r}$ is not defined for $r<1$.)

where $(v, w)$ is a point on that line. Hence the transformed curve (using $\Phi_{\mathcal{A B}}^{-1}$ ) is given by $v=u^{r} / u_{m}^{r}(b-a)+a$, and for the area of the left-hand side region in $\mathcal{R}_{r}^{+}$ cut off by this curve we get $\int_{0}^{u_{m}}\left(u^{r} / u_{m}^{r}(b-a)+a\right) d u=(b+r a) u_{m} /(r+1)$. By constraint (ii) this must be $\geq\left|\mathcal{A}_{r}^{+}\right|=\bar{v}_{r} u_{m} /(r+1)$. The latter equality follows from (14) and the definition of $\bar{v}_{r}$. Hence $b+r a \geq \bar{v}_{r}$. Now suppose

$$
\left(\bar{v}_{r} /(r+1), u_{m}^{r} /(r+1)\right) \notin \mathcal{C}^{+} .
$$

Then by (28)

$$
\bar{v}_{r} /(r+1)>\left(u_{m}^{r} /(r+1)\right) / u_{m}^{r}(b-a)+a
$$

and thus $\bar{v}_{r}>b+r a$, a contradiction. Therefore $\Delta \subseteq \mathcal{C}^{+}$. Consequently $\mathcal{C}^{+}=\Delta$ and the inequality (25) follows. Analogously we find a set $\mathcal{C}^{-}$and inequality (26) for the left-hand rectangle $\mathcal{R}_{r}^{-}$.

To verify (27) we can compute $\left|\mathcal{S}_{r}^{+}\right|$in the same way as equation (15). We only have to replace the vertex $\left(v^{+}, u_{e}^{r}\right)$ by the vertex $\left(\bar{v}_{r} /(r+1), u_{m}^{r} /(r+1)\right)$, i.e., we have to replace $v^{+}$by $\left(1-F(\mu)\left(\int f\right) /\left(u_{m}(r+1)\right)=\left(1-F(\mu)\left|\mathcal{A}_{r}\right| / u_{m}\right.\right.$ and $u_{e}$ by $u_{m} /(r+1)^{1 / r}$ in equation (15). Since $\left|\mathcal{S}_{r}\right|=\left|\mathcal{S}_{r}^{-}\right|+\left|\mathcal{S}_{r}^{+}\right|$, (27) follows by a straightforward computation.

\section{4. $T_{c}$-CONCAVE DISTRIBUTIONS}

The envelopes and squeezes introduced in the previous section can be used to derive general upper and lower bounds that hold for all $T_{c}$-concave distributions. It is a straightforward computation. Universal majorizing and minimizing functions for the density function are obtained by applying the transformation $\Phi_{\mathcal{B G}} \circ \Phi_{\mathcal{A B}}$ (see (13)) on the respective boundaries of $\mathcal{R}_{r}$ and $\mathcal{Q}_{r}$ (Theorem[7), and $\mathcal{S}_{r}$ (Theorem 11). We only give the upper bounds that do not require the evaluation of the c.d.f. at the mode. All other bounds can be derived in completely the same way.

Theorem 12. Let $f(x)$ be a $T_{c}$-concave density function with $c=-\frac{r}{r+1}, r>0$, and mode $\mu$. Let $u_{m}=\sqrt[r+1]{f(\mu)}$ (as in Theorem 7) and $x_{m}=\int f / f(\mu)$. Define $\theta:(0, \infty) \rightarrow(0, \infty)$ and $\psi:(0, \infty) \rightarrow(0, \infty)$ by

$$
\theta^{-1}(x)=x \frac{1-x^{r}}{1-x} \quad \text { and } \quad \psi(x)=\theta^{r+1}\left(x r f(\mu) / \int f\right) .
$$


Let

$$
h(x)= \begin{cases}f(\mu) & \text { for }-x_{m} \leq x-\mu \leq x_{m}, \\ f(\mu) / \psi(|x-\mu|) & \text { otherwise }\end{cases}
$$

Then $h(x) \geq f(x)$ for all $x$.

Proof. First notice that $\theta^{-1}:(0, \infty) \rightarrow(0, \infty)$ is strictly monotonically increasing and $\theta^{-1}(0)=0$. Hence its inverse map $\theta$ exists and is well defined. This can easily be seen, since $\left(\theta^{-1}\right)^{\prime}(x)=\left(1-(r+1) x^{r}+r x^{r+1}\right) /(x-1)^{2}$ is positive for all $x>0$, because the denominator is always nonnegative and the numerator $1-(r+1) x^{r}+r x^{r+1}$ is 0 for $x=1$, strictly monotonically decreasing for $x \in(0,1)$ and strictly monotonically increasing for $x>1$ (use its derivative).

Now $\Phi_{\mathcal{B G}} \circ \Phi_{\mathcal{A B}}:(V, U) \mapsto\left(V / U^{r}+\mu, U^{r+1}\right)$ maps the bounding curve of $\mathcal{Q}_{r}$ into the graph of a hat function for the density $f$. The straight line through the upper edge of $\mathcal{Q}_{r}$ in Theorem 7 is mapped into $y=u_{m}^{r+1}=f(\mu)$. The bounding curve $v(u)=\left(\int f\right) /\left(r u_{m}\right) \frac{\left(u / u_{m}\right)^{r}-1}{\left(u / u_{m}\right)-1}$ is mapped into the curve given by $y=u^{r+1}$ and

$$
\begin{aligned}
x(y)-\mu & =v(u) / u^{r}=\left(\int f\right) /\left(r u_{m}^{r+1}\right) \frac{1-\left(u_{m} / u\right)^{r}}{1-\left(u_{m} / u\right)}\left(u_{m} / u\right) \\
& =\left(\int f\right) /\left(r u_{m}^{r+1}\right) \theta^{-1}\left(u_{m} / u\right)=\left(\int f\right) /(r f(\mu)) \theta^{-1}(\sqrt[r+1]{f(\mu) / y}) .
\end{aligned}
$$

Hence $\sqrt[r+1]{y(x)}=\sqrt[r+1]{f(\mu)} / \theta\left((x-\mu) r f(\mu) / \int f\right)$ and $y(x)=f(\mu) / \psi(x-\mu)$ for $x \geq$ $\mu$. Since the right upper vertex of $\mathcal{Q}_{r}$ is given by $\left(\left(\int f\right) / u_{m}, u_{m}\right)$, the corresponding vertex in the graph of the hat function is at $x=v / u^{r}=\left(\int f\right) / f(\mu)=x_{m}$. We get an analogous result for the left-hand bounding curve. Thus the result follows.

This upper bound can be applied to sample from the $T_{c}$-concave distribution. For sampling from the hat distribution the inverse-of- $f$ method [7 $\S I V .6 .3$, p.178] can be used. However, this requires an appropriate hat function for $\sqrt[r+1]{\theta^{-1}(x)}$ similar to those in Theorem 10.

Remark. The bound of Theorem 12 is optimal in the sense that any improvement requires more information about the distribution besides the location of the mode and the c.d.f. at the mode.

Remark. By our construction of the hat function in Theorem 12, the rejection constant $\alpha$ follows immediately from equation (20), i.e.,

$$
\alpha=\alpha_{\text {univ }}(r)=\frac{r+1}{r}(\gamma+\psi(r+1)) .
$$

(To be more precise: this is the rejection constant when we use $F(\mu)$ for constructing the hat.) Another way to construct a mountain-table shaped hat for a $T_{c}$-concave density is by means of transformed density rejection with the mode of the density and two points on either side of it as construction points. By the theory developed by Derflinger it is possible to optimize such a hat function for a given density [4]. The rejection constant $\alpha$ is then bounded from above for a given $c=-r /(r+1)$ by

$$
\alpha \leq \alpha_{\mathrm{tdr}}(r)=\frac{1}{1-(1 /(r+1))^{1 / r}} .
$$

It is also shown that this bound is sharp, i.e., for any $r \geq 0$ there exists a $T_{c}$-concave density where equality holds in (32). It is interesting to note that $\alpha_{\text {univ }}(1)=\alpha_{\mathrm{tdr}}(1)$, 


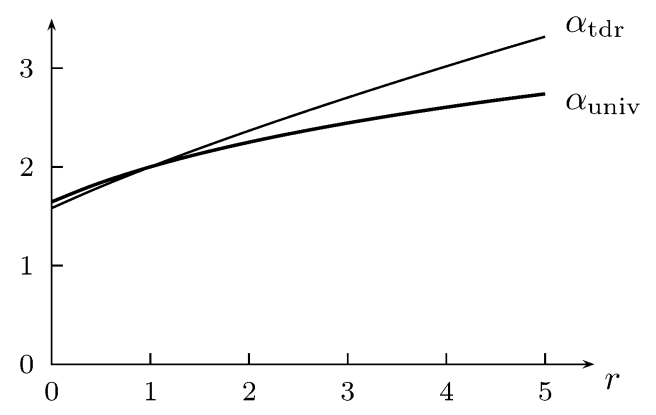

FIGURE 7. $\alpha_{\text {univ }}$ and $\alpha_{\mathrm{tdr}}$.

$\alpha_{\text {univ }}>\alpha_{\text {tdr }}$ for $r<1$, and $\alpha_{\text {univ }}<\alpha_{\text {tdr }}$ for $r>1$, see Figure 7. Consequently, the performance of the simple generator that is based on Theorem 7 is better than the worst-case performance of a more specialized generator based on TDR with three points of contact.

\section{HeAVy-TAILED Distributions}

We call a distribution a heavy-tailed $T_{c}$-concave distribution, $c<0$, if its probability density function $f(x)$ is $T_{c^{-}}$concave and $(x-\mu) f(x)^{-c}$ is monotonically increasing on $\mathbb{R}$. Notice that for densities with support not equal to $\mathbb{R}$ this only may hold if it is monotone with support $(-\infty, \mu)$ or $(\mu, \infty)$.

Again let $\mu$ be the mode of the density $f$ and define $\mathcal{A}_{r}^{+}$and $\mathcal{B}_{r}^{+}$as in $\$ 3.1$ If we have a heavy-tailed $T_{c^{-}}$-concave distribution, $\mathcal{B}_{r}^{+}$is convex and contains the triangle with vertices at $(0,0),\left(0, u_{m}^{r}\right)$ and $\left(v^{+}, 0\right)$, where $u_{m}$ and $v^{+}$are defined by (11). Moreover if $\mathcal{B}_{r}^{+}$contains a boundary point $\left(v_{b}, u_{b}^{r}\right), v_{b}>0$, then $\mathcal{B}_{r}^{+}$also contains the quadrangle with vertices at $(0,0),\left(0, u_{m}^{r}\right),\left(v_{b}, u_{b}^{r}\right)$ and $\left(v_{b}, 0\right)$. By the transformation $\Phi_{\mathcal{A B}}^{-1}$ it is mapped into a four-sided region in $\mathcal{A}_{r}$ of area

$$
\int_{0}^{v_{b}}\left(u_{m}^{r}+v\left(u_{b}^{r}-u_{m}^{r}\right) / v_{b}\right)^{1 / r} d v=v_{b} \frac{r}{r+1} \frac{u_{b}^{r+1}-u_{m}^{r+1}}{u_{b}^{r}-u_{m}^{r}}
$$

which cannot exceed $\left|\mathcal{A}_{r}^{+}\right|$. Thus we find, analogously to 33.1 that

$$
v_{b} \leq(1-F(\mu))\left(\int f\right) \frac{1}{r u_{m}} \frac{\left(u_{b} / u_{m}\right)^{r}-1}{\left(u_{b} / u_{m}\right)^{r+1}-1} .
$$

An analogous (lower) bound can be deduced for a boundary point $\left(v_{b}, u_{b}^{r}\right)$ with $v_{b}<0$. Thus we have the following universal envelopes.

Theorem 13. Let $f(x)$ be a heavy-tailed $T_{c}$-concave density function with $c=$ $-\frac{r}{r+1}, r>0$, and mode $\mu$. Let $F$ denote the c.d.f. of the distribution, and let

$$
\begin{aligned}
& \mathcal{R}_{r}=\left\{(v, u): v_{l}(u) \leq v \leq v_{r}(u), 0 \leq u \leq u_{m}\right\}, \\
& \mathcal{Q}_{r}=\left\{(v, u):-v_{m}(u) \leq v \leq v_{m}(u), 0 \leq u \leq u_{m}\right\},
\end{aligned}
$$

where

$$
\begin{array}{ll}
u_{m}=\sqrt[r+1]{f(\mu)}, & v_{m}(u)=\left(\int f\right) /\left(r u_{m}\right) \frac{\left(u / u_{m}\right)^{r}-1}{\left(u / u_{m}\right)^{r+1}-1} \\
v_{l}(u)=-F(\mu) v_{m}(u), & v_{r}(u)=(1-F(\mu)) v_{m}(u) .
\end{array}
$$


Then $\mathcal{A}_{r} \subset \mathcal{R}_{r} \subset \mathcal{Q}_{r}$ and

$$
\left|\mathcal{R}_{r}\right|=-\frac{1}{r}\left(\gamma+\psi\left(\frac{1}{r+1}\right)\right)\left|\mathcal{A}_{r}\right| \quad \text { and } \quad\left|\mathcal{Q}_{r}\right|=2\left|\mathcal{R}_{r}\right|,
$$

where $\gamma=0.577216 \ldots$ denotes Euler's (gamma) constant and $\psi(z)=\Gamma^{\prime}(z) / \Gamma(z)$ is the digamma function (also known as Euler's psi function).

Proof. By the above considerations it remains to verify (37). From (36) and (14) we get

$$
\begin{aligned}
\left|\mathcal{R}_{r}\right| & =\left|\mathcal{R}_{r}^{+}\right|+\left|\mathcal{R}_{r}^{-}\right|=\int_{0}^{u_{m}}\left(\int f\right) /\left(r u_{m}\right) \frac{\left(u / u_{m}\right)^{r}-1}{\left(u / u_{m}\right)^{r+1}-1} d u \\
& =\int_{0}^{u_{m}}\left|\mathcal{A}_{r}\right| \frac{r+1}{r} \frac{\left(u / u_{m}\right)^{r}-1}{\left(u / u_{m}\right)^{r+1}-1} \frac{1}{u_{m}} d u=\left|\mathcal{A}_{r}\right| \frac{r+1}{r} \int_{0}^{1} \frac{z^{r}-1}{z^{r+1}-1} d z .
\end{aligned}
$$

Substituting $t$ for $z^{r+1}$ yields

$$
-\frac{1}{r+1} \int_{0}^{1} \frac{t^{-r /(r+1)}-1}{t-1} d t=-\frac{1}{r+1}\left(\gamma+\psi\left(\frac{1}{r+1}\right)\right)
$$

for the integral, where the last equality follows from formula 8.361(7) in [12, p.952]. Thus the result follows.

The envelopes $\mathcal{R}_{r}$ and $\mathcal{Q}_{r}$ in Theorem 13 are optimal. Figure 8 illustrates the situation for Student's distribution with $\nu=1 / 3$ degrees of freedom. Notice that $\left|\mathcal{R}_{r}\right| /\left|\mathcal{A}_{r}\right|$ converges to 1 when $r \rightarrow \infty$ (see Figure 9).

Generating points uniformly over $\mathcal{R}_{r}$ requires an appropriate enveloping region. A rectangle is the most convenient one. Notice that $\frac{\left(u / u_{m}\right)^{r}-1}{\left(u / u_{m}\right)^{r+1}-1}$ is maximized at $u=0$ in $\left[0, u_{m}\right]$. Thus we find the following (minimal) bounding rectangle for $\mathcal{R}_{r}$.

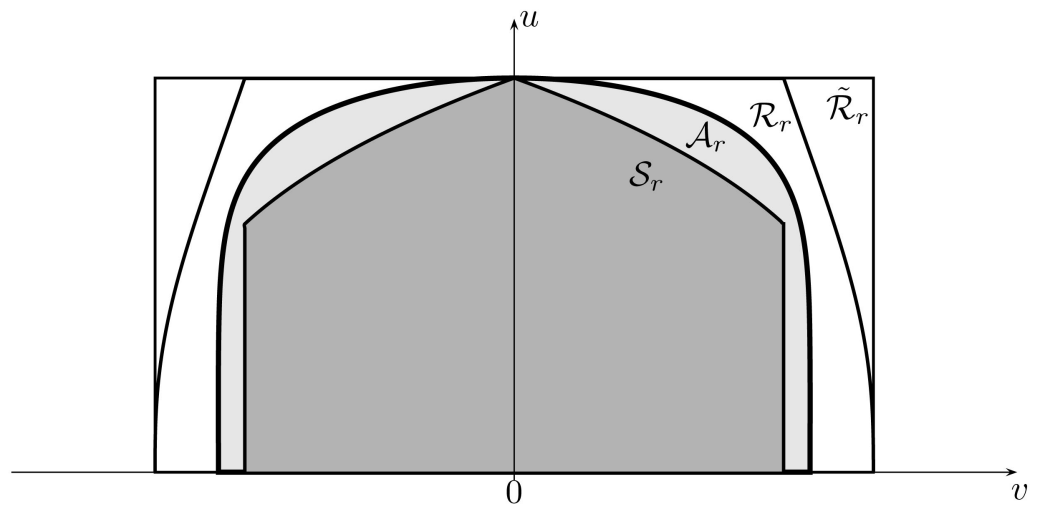

FiguRE 8. $\mathcal{A}_{r}$ and universal bounding envelopes $\mathcal{R}_{r}$ and $\tilde{\mathcal{R}}_{r}$, and universal squeeze $\mathcal{S}_{r}$ for Student's distribution with $\nu=1 / 3$ degree of freedom $(r=3)$. 


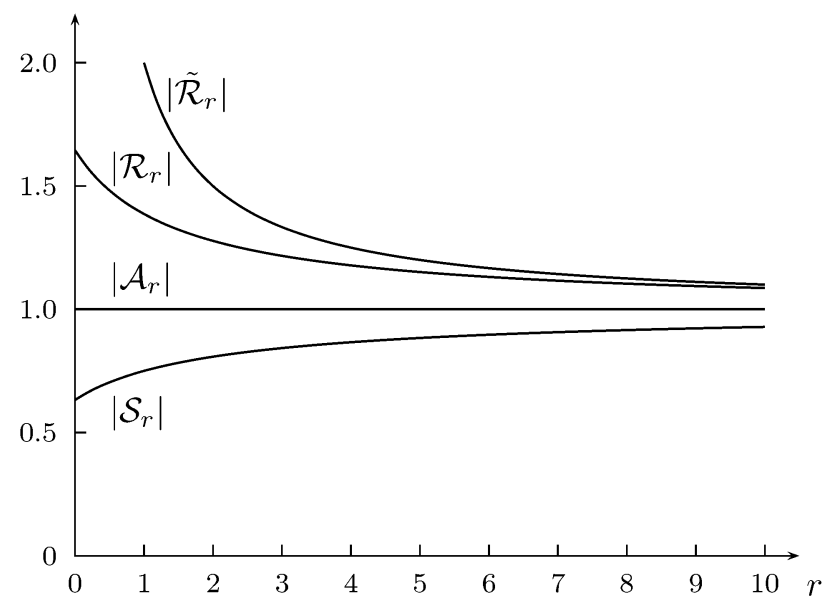

FiguRE 9. Respective ratios of $\left|\tilde{\mathcal{R}}_{r}\right|,\left|\mathcal{R}_{r}\right|$ and $\left|\mathcal{S}_{r}\right|$ to $\left|\mathcal{A}_{r}\right|$ as functions of $r$ for heavy-tailed distributions.

Theorem 14. Let $f(x)$ be a heavy-tailed $T_{c}$-concave density function with $c=$ $-\frac{r}{r+1}, r>0$, and mode $\mu$. Let $F$ denote the c.d.f. of the distribution, and let

$$
\begin{aligned}
& \tilde{\mathcal{R}}_{r}=\left\{(v, u): \tilde{v}_{l} \leq v \leq \tilde{v}_{r}, 0 \leq u \leq u_{m}\right\}, \\
& \tilde{\mathcal{Q}}_{r}=\left\{(v, u):-\tilde{v}_{m} \leq v \leq \tilde{v}_{m}, 0 \leq u \leq u_{m}\right\},
\end{aligned}
$$

where

$$
\begin{array}{ll}
u_{m}=\sqrt[r+1]{f(\mu)}, & \tilde{v}_{m}=\left(\int f\right) /\left(r u_{m}\right), \\
\tilde{v}_{l}=-F(\mu) \tilde{v}_{m}, & \tilde{v}_{r}=(1-F(\mu)) \tilde{v}_{m} .
\end{array}
$$

Then $\mathcal{A}_{r} \subset \tilde{\mathcal{R}}_{r} \subset \tilde{\mathcal{Q}}_{r}$ and

$$
\left|\tilde{\mathcal{R}}_{r}\right|=\frac{r+1}{r}\left|\mathcal{A}_{r}\right| \quad \text { and } \quad\left|\tilde{\mathcal{Q}}_{r}\right|=2\left|\tilde{\mathcal{R}}_{r}\right| .
$$

Although the envelope $\tilde{\mathcal{R}}_{r}$ is not optimal for small $r$, we find that $\left|\tilde{\mathcal{R}}_{r}\right|$ converges to $\left|\mathcal{R}_{r}\right|$ for $r \rightarrow \infty$ (see Figure 9). In particular, we have $\left|\tilde{\mathcal{R}}_{r}\right|<1.1\left|\mathcal{R}_{r}\right|$ for $r \geq 4$. So it is only necessary to find sophisticated generators for the distribution with density proportional to $\frac{z^{r}-1}{z^{r+1}-1}$ if $r$ is small. For $r=1, \frac{z^{r}-1}{z^{r+1}-1}$ reduces to $1 /(z+1)$.

We also can find a universal squeeze for heavy-tailed distributions.

Theorem 15. Let $f(x)$ be a heavy-tailed $T_{c}$-concave probability density function with $c=-\frac{r}{r+1}, r>0$, and mode $\mu$. If $F(\mu)$ is given, where $F$ denotes the c.d.f. of the distribution, then there exists a set $\mathcal{S}_{r}=\mathcal{S}_{r}(f)$ such that $\mathcal{S}_{r} \subset \mathcal{A}_{r}(f)$. We have $(V, U) \in \mathcal{S}_{r}$ if and only if

$$
\bar{v}_{l}\left(u_{m}^{r}-U^{r}\right) \leq r u_{m}^{r} V \leq \bar{v}_{r}\left(u_{m}^{r}-U^{r}\right)
$$

and

$$
\bar{v}_{l} /(r+1) \leq V \leq \bar{v}_{r} /(r+1),
$$


where $u_{m}$ is as defined in Theorem 13 and $\bar{v}_{r}=(1-F(\mu))\left(\int f\right) / u_{m}$ and $\bar{v}_{l}=$ $-F(\mu)\left(\int f\right) / u_{m}$. Moreover,

$$
\left|\mathcal{S}_{r}\right|=\left|\mathcal{A}_{r}\right|\left(1-1 /(r+1)^{(r+1) / r}\right) .
$$

Proof. Since every heavy-tailed $T_{c}$-concave distribution is $T_{c}$-concave, inequality (41) follows immediately from Theorem 11 Furthermore, notice that

$$
\left(\bar{v}_{r} /(r+1), u_{m}^{r} /(r+1)\right)
$$

is contained in the transformed squeeze region (in $\mathcal{B}_{r}^{+}$, see proof of Theorem 11). Moreover, if $\mathcal{B}_{r}^{+}$contains a boundary point $\left(v_{b}, u_{b}^{r}\right)$, then $\mathcal{B}_{r}^{+}$also contains the quadrangle with vertices at $(0,0),\left(0, u_{m}^{r}\right),\left(v_{b}, u_{b}^{r}\right)$ and $\left(v_{b}, 0\right)$ (as shown above). Thus (42) follows.

To verify (43) we compute

$$
\int_{0}^{v_{b}}\left(u_{m}^{r}+w / v_{b}\left(u_{b}^{r}-u_{m}^{r}\right)\right)^{1 / r} d w=\frac{r}{r+1} u_{m} v_{b} \frac{\left(u_{b} / u_{m}\right)^{r+1}-1}{\left(u_{b} / u_{m}\right)^{r}-1} .
$$

Using the vertex $\left(\bar{v}_{r} /(r+1), u_{m}^{r} /(r+1)\right)$ for $\left(v_{b}, u_{b}^{r}\right)$ gives $v_{b}=\bar{v}_{r} /(r+1)=$ $(1-F(\mu)) \int f /\left(u_{m}(r+1)\right)=(1-F(\mu))\left|\mathcal{A}_{r}\right| / u_{m}$ and $u_{b}=u_{m} /(r+1)^{1 / r}$. Then (44) reduces to $(1-F(\mu))\left|\mathcal{A}_{r}\right|\left(1-1 /(r+1)^{(r+1) / r}\right)$, and the result follows from the analogous considerations for $\mathcal{B}_{r}^{-}$.

Remark. If $f$ is the probability density function of a heavy-tailed $T_{c}$-concave distribution for a particular $c$, then this does not hold for any other $c^{\prime} \neq c$. Indeed, $(x-\mu) f(x)^{-c}$ is increasing and bounded only if $f$ behaves asymptotically as $x^{1 / c}$ for $x \rightarrow \infty$. Thus for any $c^{\prime} \neq c,(x-\mu) f(x)^{-c}$ either converges to 0 or is unbounded.

Now let $f$ be a differentiable function. [3] calls the largest $c \leq 0$ with $\left(T_{c}(f(x))\right)^{\prime \prime}$ $\leq 0$ the $T_{c}$-concavity of $f$ at $x$, denoted by tc $f(x)$. (We set tc $\left.f(x)\right)=-\infty$, if no such $c$ exists.) Then a monotonically decreasing density is heavy-tailed $T_{c}$-concave if and only if $f$ is $T_{c^{-}}$concave and $c=\liminf _{x \rightarrow \infty} \operatorname{tc}_{f}(x)>-1$. An analogous characterization holds for monotonically increasing and symmetric densities. Some standard distributions (e.g., $t$-distributions) have the property that $\operatorname{tc}_{f}(x)$ is monotonically decreasing on $(\mu, \infty)$ and increasing in $(-\infty, \mu)$.

\section{Discrete Distributions}

[8] has introduced a universal hat for discrete log-concave distributions that can be applied for the design of a black box algorithm. In [19] a faster and more generally applicable algorithm based on the ratio-of-uniforms method has been introduced. Therefore it is not amazing that a version of Theorem 10 can be found for discrete distributions. However, some modifications are necessary.

A discrete distribution with probability vector $p_{i}$, with support $I \subseteq \mathbb{Z}$, is called $T$-concave if

$$
T\left(p_{i}\right) \geq \frac{1}{2}\left(T\left(p_{i-1}\right)+T\left(p_{i+1}\right)\right) \quad \text { for all } i \in I .
$$

For log-concave distributions we have $T(x)=\log (x)$ and $p_{i}^{2} \geq p_{i-1} p_{i+1}$. Obviously $p_{i}$ is unimodal. Denote its mode by $\mu$. For the following, assume that $p_{i}$ is $T_{c^{-}}$ concave with $c=-r /(r+1)$ for an $r>0$. Let

$$
f_{p}(x)= \begin{cases}p_{\lfloor x\rfloor} & \text { for }\lfloor x\rfloor \in I \\ 0 & \text { otherwise }\end{cases}
$$


where $\lfloor x\rfloor$ denotes the largest integer not greater than $x$. Since $f_{p}$ is a step function, $\mathcal{B}_{r}\left(f_{p}\right)$ cannot be convex, where $\mathcal{B}_{r}$ is defined by (12). The outer vertices of $\mathcal{B}_{r}$ (its "spikes") are given by

$$
\left((i+1-\mu) p_{i}^{r /(r+1)}, p_{i}^{r /(r+1)}\right)
$$

for all $i \geq \mu$, and

$$
\left((i-\mu) p_{i}^{r /(r+1)}, p_{i}^{r /(r+1)}\right)
$$

for all $i \leq \mu$, with $i \in I \subseteq \mathbb{Z}$ (use the transformation $\Phi_{\mathcal{B G}}$ from (13)). Let $\left(v^{+}, u_{e}^{r}\right)$ be the right extremal point of $\mathcal{B}_{r}$ and let $\Delta_{\mathcal{B}}$ denote the triangle with vertices at $(0,0),\left(0, u_{m}^{r}\right)$ and $\left(v^{+}, u_{e}^{r}\right)$ in $\mathcal{B}_{r}^{+}=\left\{\left(v, u^{r}\right) \in \mathcal{B}_{r}: v \geq 0\right\}$. Notice that by inequality (45) there are no outer vertices of $\mathcal{B}_{r}$ in the interior of $\Delta_{\mathcal{B}}$. Let $\Delta_{\mathcal{A}}$ be the image of $\Delta_{\mathcal{B}}$ under the transformation $\Phi_{\mathcal{A B}}^{-1}$.

Lemma 16. $\left|\Delta_{\mathcal{A}}\right| \leq\left|\mathcal{A}_{r}^{+}\right|$, where $\mathcal{A}_{r}^{+}=\left\{(v, u) \in \mathcal{A}_{r}: v \geq 0\right\}$.

Proof. For the proof we again need the transformation (13). Denote the images of $\Delta_{\mathcal{B}}$ under the respective transformations $\Phi_{\mathcal{B G}}$ and $\Phi_{\mathcal{G} \mathcal{T}} \circ \Phi_{\mathcal{B G}}$ by $\Delta_{\mathcal{G}}$ and $\Delta_{\mathcal{T}}$. The latter transformation $\Phi_{\mathcal{G} \mathcal{T}} \circ \Phi_{\mathcal{B G}}$ maps straight lines into straight lines (see the proof of Theorem 6 ). Thus $\Delta_{\mathcal{T}}$ is a convex (unbounded) polygon. Moreover, the edge $(0,0)\left(0, u_{m}^{r}\right)$ is mapped into the $y$-axis.

Now assume $u_{e}=0$. Then $\Delta_{\mathcal{T}}$ is bounded only by the images of the edges $(0,0)\left(0, u_{m}^{r}\right)$ and $\left(0, u_{m}^{r}\right)\left(v^{+}, u_{e}^{r}\right)$. Since both are straight lines, they are subsets of $\mathcal{T}_{c}, c=-r /(r+1)$, by inequality (45). Hence $\Delta_{\mathcal{T}} \subseteq \mathcal{T}_{c}$, and so $\Delta_{\mathcal{A}} \subseteq \mathcal{A}_{r}^{+}$, and the proposition follows.

If $u_{e}>0$, the edge $\left(0, u_{m}\right)\left(v^{+}, u_{e}^{r}\right)$ of $\Delta_{\mathcal{B}}$ is contained in the quadrangle $Q_{\mathcal{B}}$ with vertices $\left(0, u_{m}^{r}\right),\left(u_{m}^{r}, u_{m}^{r}\right),\left(v^{+}, u_{e}^{r}\right)$ and $\left(v^{+}-u_{e}^{r}, u_{e}^{r}\right)$, see Figure 10 (1.h.s.). Using $\Phi_{\mathcal{G} \mathcal{T}} \circ \Phi_{\mathcal{B G}}, Q$ is mapped into a set $Q_{\mathcal{T}}$ which again is a quadrangle by Theorem 6 see Figure 10 (r.h.s.). Notice that for the areas we get $\left|Q_{\mathcal{T}} \cap \Delta_{\mathcal{T}}\right|=\left|Q_{\mathcal{T}}\right| / 2$ and $\left|Q_{\mathcal{T}} \cap \mathcal{T}_{c}\right| \geq\left|Q_{\mathcal{T}}\right| / 2$. Figure 10 shows the "worst case", where equality holds in (45) for all $i$ between the mode and the point that corresponds to the extremal point $\left(v^{+}, u_{e}^{r}\right)$. Now we can use the transformation $\Phi_{\mathcal{G} \mathcal{T}}^{-1}$ to map $Q_{\mathcal{T}}$ into a set $Q_{\mathcal{G}}$. We have to show that $\left|\Delta_{\mathcal{G}}\right| \leq\left|\mathcal{G}^{+}\right|$, where $\mathcal{G}^{+}=\{(x, y) \in \mathcal{G}: y \leq f(x)\}$. Then the result follows because the Jacobian of $\Phi_{\mathcal{B G}} \circ \Phi_{\mathcal{A B}}$ is constant, and thus $\left|\Delta_{\mathcal{A}}\right| \leq\left|\mathcal{A}^{+}\right|$ follows as proposed.

Now let $Q_{\mathcal{G}}$ denote the image of $Q_{\mathcal{T}}$ under the transformation $\Phi_{\mathcal{G} \mathcal{T}}^{-1}$. To verify that $\left|\Delta_{\mathcal{G}}\right| \leq\left|\mathcal{G}^{+}\right|$, it is sufficient to show that the area of the image of $Q_{\mathcal{T}} \cap \mathcal{T}_{c}$ under the transformation $\Phi_{\mathcal{G} \mathcal{T}}^{-1}$, i.e., $Q_{\mathcal{G}} \cap \mathcal{G}$, is larger than the area of the image of the triangle $Q_{\mathcal{T}} \cap \Delta_{\mathcal{T}}$, i.e., $Q_{\mathcal{G}} \cap \Delta_{\mathcal{G}}$. The absolute value of the Jabobian of $\Phi_{\mathcal{G} \mathcal{T}}^{-1}$ is given by $1 / c(-y)^{1 / c-1}$, which is decreasing for decreasing $y$, since $y<0$. Hence we immediately get (see r.h.s. of Figure 10) $\left|Q_{\mathcal{G}} \cap \Delta_{\mathcal{G}}\right| \leq\left|Q_{\mathcal{G}}\right| / 2$. To see that $\left|Q_{\mathcal{G}} \cap \mathcal{G}\right|>\left|Q_{\mathcal{G}}\right| / 2$, notice that we can split $Q_{\mathcal{T}}$ horizontally along the upper edges of the bars in Figure 10. In each of these parts the shaded region that belongs to $\mathcal{T}_{c}$ has at least the same area as its complement outside. Moreover, the shaded part is more concentrated towards the $x$-axis than its complement. Thus, if we look at the images of this region and of its complement under $\Phi_{\mathcal{G} \mathcal{T}}^{-1}$, the area of the former is always greater than the area of the latter and thus $\left|Q_{\mathcal{G}} \cap \mathcal{G}\right|>\left|Q_{\mathcal{G}}\right| / 2$. Hence $\left|Q_{\mathcal{G}} \cap \mathcal{G}\right|>\left|Q_{\mathcal{G}} \cap \Delta_{\mathcal{G}}\right|$, and the result follows. 

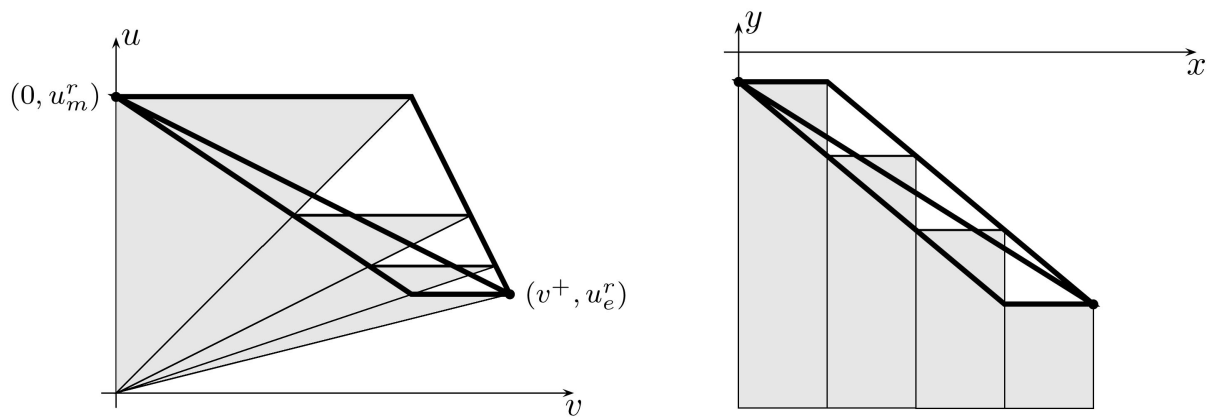

FiguRE 10. Quadrangle $Q_{\mathcal{B}}$ for $\mathcal{B}_{r}$ (l.h.s.) and the corresponding quadrangle $Q_{\mathcal{T}}$ for $\mathcal{T}_{c}$ (r.h.s.)

It is obvious that an analogous result holds for $\mathcal{A}_{r}^{-}$, and we arrive at the following proposition analogously to our considerations of Theorem 7 . Notice that $\sup _{i<\mu} p_{i}=p_{\mu-1}$.

Theorem 17. Let $p_{i}, i \in \mathbb{Z}$, be a $T_{c}$-concave probability vector with $c=-r /(r+1)$, $r>0$, and mode $\mu$. Let $F$ denote the c.d.f. of the distribution, and let

$$
\begin{array}{ll}
u_{l}=\sqrt[r+1]{p_{\mu-1}}, & v_{l}(u)=\left(\sum p_{i}\right) /\left(r u_{l}\right) \frac{\left(u / u_{l}\right)^{r}-1}{\left(u / u_{l}\right)-1}, \\
u_{r}=\sqrt[r+1]{p_{\mu}}, & v_{r}(u)=\left(\sum p_{i}\right) /\left(r u_{r}\right) \frac{\left(u / u_{r}\right)^{r}-1}{\left(u / u_{r}\right)-1},
\end{array}
$$

where we set $v_{l}(u)=0$ if $u_{l}=0$. Let $\mathcal{R}_{r}=\mathcal{R}_{r}^{-} \cup \mathcal{R}_{r}^{+}$with

$$
\begin{aligned}
& \mathcal{R}_{r}^{-}=\left\{(v, u):-F(\mu) v_{l}(u) \leq v \leq 0,0 \leq u \leq u_{l}\right\}, \\
& \mathcal{R}_{r}^{+}=\left\{(v, u): 0 \leq v \leq(1-F(\mu)) v_{r}(u), 0 \leq u \leq u_{r}\right\},
\end{aligned}
$$

and $\mathcal{Q}_{r}=\mathcal{Q}_{r}^{-} \cup \mathcal{Q}_{r}^{+}$with

$$
\begin{aligned}
& \mathcal{Q}_{r}^{-}=\left\{(v, u): v_{l}(u) \leq v \leq 0,0 \leq u \leq u_{l}\right\} \\
& \mathcal{Q}_{r}^{+}=\left\{(v, u): 0 \leq v \leq v_{r}(u), 0 \leq u \leq u_{r}\right\}
\end{aligned}
$$

Then $\mathcal{A}_{r} \subset \mathcal{R}_{r} \subset \mathcal{Q}_{r}$ and

$$
\left|\mathcal{R}_{r}\right|=\frac{r+1}{r}(\gamma+\psi(r+1))\left|\mathcal{A}_{r}\right| \quad \text { and } \quad\left|\mathcal{Q}_{r}\right|=2\left|\mathcal{R}_{r}\right|,
$$

where $\gamma=0.577216 \ldots$ denotes Euler's (gamma) constant and $\psi(z)=\Gamma^{\prime}(z) / \Gamma(z)$ is the digamma function (also known as Euler's psi function).

For sampling uniformly from $\mathcal{Q}_{r}$ and $\mathcal{R}_{r}$, the envelopes from Theorem 10 can be used. It is straightforward to find a universal squeeze analogously to continuous distributions. Furthermore, bounds for discrete heavy-tailed $T_{c}$-concave distributions exist.

\section{REFERENCES}

[1] Ahrens, J. H. (1993). Sampling from general distributions by suboptimal division of domains. Grazer Math. Berichte 319, 20 pp.

[2] Ahrens, J. H. (1995). A one-table method for sampling from continuous and discrete distributions. Computing 54(2), 127-146. MR 96a:65011

[3] Derflinger, G. (2000). private communication. 
[4] Derflinger, G., W. Hörmann, and J. Leydold (2002). Universal methods of nonuniform random variate generation. in preparation.

[5] Devroye, L. (1984a). On the use of probability inequalities in random variate generation. $J$. Stat. Comput. Simulation 20, 91-100. MR 87g:65009

[6] Devroye, L. (1984b). A simple algorithm for generating random variates with a log-concave density. Computing 33(3-4), 247-257. MR 86d:65019

[7] Devroye, L. (1986). NonUniform Random Variate Generation. New-York: Springer-Verlag. MR 87i:65012

[8] Devroye, L. (1987). A simple generator for discrete log-concave distributions. Computing 39, 87-91. MR 89f:65007

[9] Dieter, U. (1989). Mathematical aspects of various methods for sampling from classical distributions. In E. A. Mc Nair, K. J. Musselman, and P. Heidelberger (Eds.), Proc. 1989 Winter Simulation Conf., pp. 477-483.

[10] Evans, M. and T. Swartz (1998). Random variable generation using concavity properties of transformed densities. Journal of Computational and Graphical Statistics 7(4), 514-528.

[11] Gilks, W. R. and P. Wild (1992). Adaptive rejection sampling for Gibbs sampling. Applied Statistics 41(2), 337-348.

[12] Gradshteyn, I. S. and I. M. Ryzhnik (1994). Table of Integrals, Series, and Products (5th ed.). Academic Press. MR 94g:00008

[13] Hörmann, W. (1995). A rejection technique for sampling from T-concave distributions. ACM Trans. Math. Software 21(2), 182-193. MR 96b:65018

[14] Hörmann, W. and G. Derflinger (1996). Rejection-inversion to generate variates from monotone discrete distributions. ACM TOMACS 6(3), 169-184.

[15] Hörmann, W. and G. Derflinger (1997). An automatic generator for a large class of unimodal discrete distributions. In A. R. Kaylan and A. Lehmann (Eds.), ESM 97, pp. 139-144.

[16] Kinderman, A. J. and F. J. Monahan (1977). Computer generation of random variables using the ratio of uniform deviates. ACM Trans. Math. Software 3(3), 257-260.

[17] Leydold, J. (2000a). Automatic sampling with the ratio-of-uniforms method. ACM Trans. Math. Software 26(1), 78-98.

[18] Leydold, J. (2000b). A note on transformed density rejection. Computing 65(2), 187-192. MR 2002e: 65016

[19] Leydold, J. (2001). A simple universal generator for continuous and discrete univariate Tconcave distributions. ACM Trans. Math. Software 27(1), 66-82.

[20] Stadlober, E. (1989). Sampling from Poisson, binomial and hypergeometric distributions: Ratio of uniforms as a simple and fast alternative. Number 303 in Bericht der MathematischStatistischen Sektion in der Forschungsgesellschaft Joanneum-Graz. MR 91b:65008

[21] Wakefield, J. C., A. E. Gelfand, and A. F. M. Smith (1991). Efficient generation of random variates via the ratio-of-uniforms method. Statist. Comput. 1(2), 129-133.

University of Economics and Business Administration, Department for Applied Statistics and Data Processing, Augasse 2-6, A-1090 Vienna, Austria

E-mail address: Josef.Leydold@statistik.wu-wien.ac.at

URL: http://statistik.wu-wien.ac.at/staff/leydold/ 